\title{
High-resolution melting analysis for SNP genotyping and mapping in tetraploid alfalfa (Medicago sativa $\mathrm{L}_{\text {.) }}$
}

\author{
Yuanhong Han · Dong-Man Khu • \\ Maria J. Monteros
}

Received: 16 December 2010/ Accepted: 12 March 2011/Published online: 29 March 2011

(C) The Author(s) 2011. This article is published with open access at Springerlink.com

\begin{abstract}
Single nucleotide polymorphisms (SNPs) represent the most abundant type of genetic polymorphism in plant genomes. SNP markers are valuable tools for genetic analysis of complex traits of agronomic importance, linkage and association mapping, genome-wide selection, map-based cloning, and marker-assisted selection. Current challenges for SNP genotyping in polyploid outcrossing species include multiple alleles per loci and lack of highthroughput methods suitable for variant detection. In this study, we report on a high-resolution melting (HRM) analysis system for SNP genotyping and mapping in outcrossing tetraploid genotypes. The sensitivity and utility of this technology is demonstrated by identification of the parental genotypes and segregating progeny in six alfalfa populations based on unique melting curve profiles due to differences in allelic composition at one or multiple loci. HRM using
\end{abstract}

Electronic supplementary material The online version of this article (doi:10.1007/s11032-011-9566-x) contains supplementary material, which is available to authorized users.

Y. Han · D.-M. Khu · M. J. Monteros ( $\square)$

Forage Improvement Division, The Samuel Roberts Noble

Foundation, 2510 Sam Noble Parkway, Ardmore,

OK 73401, USA

e-mail: mjmonteros@noble.org

Y. Han

e-mail: yhan@noble.org

D.-M. Khu

e-mail:dmkhu@noble.org a 384-well format is a fast, consistent, and efficient approach for SNP discovery and genotyping, useful in polyploid species with uncharacterized genomes. Possible applications of this method include variation discovery, analysis of candidate genes, genotyping for comparative and association mapping, and integration of genome-wide selection in breeding programs.

Keywords Alfalfa Medicago sativa .

Mapping $\cdot$ SNP $\cdot$ HRM

\section{Introduction}

Single base changes in sequence, including single nucleotide polymorphisms (SNPs) and small insertions and deletions (InDels), are the most abundant source of variation across genomes (Kwok 2001). Compared to simple sequence repeats (SSRs), SNPs are more amenable to high-throughput automated genotyping assays that allow samples to be genotyped faster and more economically (Hurley et al. 2005; Ha and Boerma 2008). SNP detection technologies have evolved from expensive, time-consuming, and labor-intensive processes to some of the most highly automated, efficient, and relatively inexpensive methods of DNA marker detection (Kwok and Chen 2003).

Polyploidy is widespread in plants and of greater evolutionary importance than originally supposed (Soltis and Soltis 1995). Autopolyploid species have more than two homologous chromosomes such that 
pairing during meiosis can occur either between randomly chosen pairs of homologous chromosomes or between more than two homologous chromosomes, resulting in a polysomic pattern of inheritance (Gallais 2003). Autopolyploid species are generally outcrossing, which maintains a high level of heterozygosity and hence greater genetic diversity compared to their diploid progenitors. Examples of autopolyploid species include alfalfa (Medicago sativa L.), sugarcane (Sacharum officinarum L.), and potato (Solanum tuberosum L.). Alfalfa is a perennial species that requires little or no nitrogen fertilizer because of its ability to perform symbiotic nitrogen fixation. Most commercially grown alfalfa species are tetraploid $(2 \mathrm{n}=4 \mathrm{x}=32)$; however, diploid species including $M$. sativa subsp. caerulea are known (McCoy and Bingham 1988). Alfalfa is partially self-incompatible and populations are quite heterozygous due to their high degree of outcrossing. Although the possible presence at many loci of more than two alleles (heterozygosity) for each individual gives perennial plants a buffering advantage to survive unstable environments (Soltis and Soltis 1995), it offers unique challenges for SNP genotyping due to the number of possible genotypes based on the number of alleles.

A number of SNP genotyping methods emerged from the availability of the Human Genome Project and vary in terms of their mechanisms for allelic discrimination, reaction formats, and method of detection (Kwok 2001). Homogenous, closed-tube methods for SNP genotyping that do not require a separation step are attractive for their simplicity and containment of amplified products. Melting curve analysis using fluorescent dyes has proven to be a highly sensitive method for mutation discovery and SNP genotyping (Liew et al. 2004; Cho et al. 2008; Smith et al. 2008). High-resolution melting (HRM) analysis is a closed-tube method for rapid analysis of genetic variation within PCR amplicons (Reed and Wittwer 2004). Upon completion of PCR in the presence of a saturating intercalating dye such as LCGreen $^{\circledR}$, which binds to double-stranded but not single-stranded DNA, the PCR product is heated while the level of fluorescence is measured. As the temperature rises and the duplex melts, dye is released and fluorescence intensity is reduced. Genetic variants with differences in base composition result in differences in melting temperature, which are detected by monitoring fluorescence during an increase in temperature and discriminated by their characteristic melting curves, visualized by a loss of fluorescence as the DNA duplex melts (Zhou et al. 2004). HRM can be performed with or without unlabeled probes. Duplexes may be formed from the two strands of a PCR amplicon or from an oligonucleotide probe and an amplicon strand (Taylor 2009). When probes are not used, the sensitivity of genotyping can be increased by using internal temperature standards and reducing the amplicon size (Seipp et al. 2007). Previous knowledge of the SNP variation present in a genotype is not required; thus HRM analysis offers unique opportunities for genotyping species with limited genomic resources. HRM has been used in genotyping in many plant species including apple (Malus domestica Borkh.) (Chagné et al. 2008), white lupin (Lupinus albus L.) (Croxford et al. 2008), almond (Prunus dulcis (Mill) D.A.Webb) (Wu et al. 2009), perennial ryegrass (Lolium perenne L.) (Studer et al. 2009), and potato (S. tuberosum) (De Koeyer et al. 2010). In this study, we developed a HRM analysis system to screen and genotype SNP markers in diploid and tetraploid alfalfa and to map SNP markers in tetraploid F1 and backcross populations.

\section{Materials and methods}

\section{Plant materials}

Parents and subsets of progeny from two diploid and four tetraploid alfalfa mapping populations were evaluated in this study (Electronic Supplementary Material Table 1). The diploid population DIP1 was derived from a cross between $M$. sativa subsp. caerulea genotypes MS-13 and MS-186. The second diploid population (DIP2) was obtained from a cross between P1-4, a M. sativa subsp. caerulea genotype, and P6-1, which is a genotype from $M$. prostrata, a diploid species closely related to alfalfa (Wang and Brummer 2010). Clonally propagated samples from a single F1 plant (F1-192) resulting from a cross between the tetraploid genotypes $M$. sativa subsp. sativa var. Chilean and M. sativa subsp. falcata var. Wisfal (Sledge et al. 2005) were backcrossed to the Chilean and the Wisfal parent to generate the backcross populations CHBC and MFBC, respectively. The tetraploid genotype Altet- 4 was crossed to the breeding 
lines NECS-141 and 95-608 to generate the two tetraploid F1 populations AL608 and ALNECS (Khu et al. 2010). Young leaf samples from all plants were harvested and processed for DNA extraction using the DNeasy plant kit (Qiagen, Valencia, CA, USA) as previously described (Zhang et al. 2008).

\section{Primer design and HRM analysis}

Twenty primer pairs targeting SNP sites were evaluated using the HRM assay (Supplementary Table 2). Three primers were developed from $M$. truncatula putative transcription factor sequences. Criteria for PCR primer design includes a predicted annealing temperature $\left(T_{\mathrm{m}}\right)$ of $58-61^{\circ} \mathrm{C}$, limited self-complementarity and poly-X, and PCR amplicon lengths of 100-150 base pairs (bp). Secondary hits were minimized by aligning primer candidates to all known Medicago sequences and eliminating primer pairs with multiple potential hits, as previously described (Kakar et al. 2008). The remaining 17 primers were developed from $M$. sativa cDNA 454 sequences using similar criteria as before except with amplicon lengths of 50-170 bp (Han et al. 2010).

All PCR reactions were performed in 384-well plates using a 9700 Thermal Cycler (Applied Biosystems, Foster City, CA, USA) in a total volume of 5 $\mu \mathrm{L}$ per well. The PCR reaction mixture consisted of $5 \mathrm{ng}$ of genomic DNA, $0.25 \mu \mathrm{M}$ of forward and reverse primer, $1 \times$ LightScanner High Sensitivity Master Mix (Idaho Technologies, Salt Lake, UT, USA) and $10 \mu \mathrm{L}$ mineral oil. Optimal annealing temperature for PCR was determined using a temperature gradient PCR ranging from 57 to $70^{\circ} \mathrm{C}$ for each primer. The experimental design included three technical replications for each sample-primer pair combination. After an initial denaturation step of 2 min at $95^{\circ} \mathrm{C}, 45 \mathrm{PCR}$ cycles were performed with $30 \mathrm{~s}$ of denaturation at $94^{\circ} \mathrm{C}$ and $30 \mathrm{~s}$ at the target annealing temperature, followed by a final hold at $4^{\circ} \mathrm{C}$. Samples were then transferred to the LightScanner 384-well system and a melting cycle was performed by increasing the temperature by $0.1^{\circ} \mathrm{C}$ $\mathrm{s}^{-1}$ from 56 to $95^{\circ} \mathrm{C}$. Melting data were analyzed using the LightScanner Software with CALL-IT 2.0 (Idaho Technologies) using the small amplicon module. The peaks resulting from the temperature standards from the two calibrators included in the High Sensitivity Genotyping Master Mix were defined on the negative first derivative plot for data calibration. The range of target melting region was defined to normalize the data. After data calibration and normalization, the samples were assigned to different genotyping groups by the LightScanner Software using hierarchical clustering based on the shape of their normalized melting curves and peaks. The sensitivity level was adjusted to discriminate all visually distinguishable groups. The clustering of genotypes into distinct groups was visualized as both normalized melting curve plots and normalized melting peaks that correspond to the normalized negative first derivative plot.

Sequencing PCR amplicons

The PCR products from the three transcription factor primers were directly sequenced using a BigDye Terminator v3.1 Cycle Sequencing Kit (Applied Biosystems) using both forward and reverse primers. The amplicons were analyzed using an ABI 3730 automated sequencer (PE-ABI, Foster City, CA, USA). The sequence data were analyzed and aligned for SNP discovery with Sequencher 4.8 (Gene Codes Corporation, Ann Arbor, MI, USA). The dosage of a heterozygous SNP in a tetraploid individual was estimated using the "Secondary as \% of Primary" of the ambiguous base reported by the software. Haplotypes were generated from sequence data using the SATlotyper software (Neigenfind et al. 2008).

\section{Mapping SNP markers}

The tetraploid populations CHBC, MFBC, and ALNECS, containing 93, 93, and 185 individuals, respectively, were used to map the polymorphic SNP markers. The predicted ability to map a marker in a certain population was determined from the HRM profile in a subset of progeny from that population. For mapping purposes, one replicate of each individual within a population was genotyped using HRM analysis. In the CHBC and MFBC populations, single-dose allele SNP markers were mapped using the Kosambi mapping function in JoinMap ${ }^{\circledR} 3.0$ software (Van Ooijen and Voorrips 2001). The composite map was obtained by joining homologous linkage groups using previously mapped SSR markers (Sledge et al. 2005) producing allelic fragments of differing sizes with the 'Combine Groups for Map 
Integration' function in JoinMap. In the ALNECS population, simplex and duplex SNP markers were mapped onto an SSR-based linkage map (Khu et al. 2010) using TetraploidMap software (Hackett and Luo 2003). SNP marker alignments on linkage group 2 were performed through BLAST queries of the amplicon sequences to the M. truncatula genome v3.5 (The International Medicago Genome Annotation Group http://www.medicago.org/genome/IMGAG/).

\section{Results}

HRM analysis for SNP detection and genotyping using small amplicons

Distinct HRM peaks were observed for each SNP genotype in the alfalfa mapping population parents, each of which is represented by a different color (Fig. 1). In all samples evaluated, the three replicates always resulted in similar curves and peaks and were assigned to the same group, indicating the consistency and reproducibility of the HRM assay. Primer MTTF063 was designed using the sequence of a putative $M$. truncatula transcription factor encoding a transducin family protein similar to the Arabidopsis At5G49430 gene to produce a PCR amplicon $111 \mathrm{bp}$ long (Supplementary Table 2). Five groups of melting curves were obtained from the diploid and tetraploid parental genotypes (Fig. 1a, b). Two groups (green and blue) exhibited the high and narrow melting curve and peaks which are typical for homozygotes. The remaining melting curve and peaks shown in gray, red, and orange are broader and shorter compared to the homozygous peaks, and correspond to the heterozygous genotypes.

In autotetraploid species, a single SNP with two variant bases (e.g. A and C) can have the following five possible genotypes: two simplex classes (ACCC and AAAC), one duplex (AACC, equivalent to the diploid AC in the shape of the melting curve) and two homozygotes (AAAA and CCCC). The melting curve profile of the diploid P1-4 (orange) indicates a heterozygous allelic composition at the target SNP site (Fig. 1a). P6-1 (green), MS-13 (blue), and MS186 (blue) exhibit a homozygous melting profile. The melting profile of the tetraploid genotype Wisfal (blue) suggests a homozygote class, while 95-608 is clustered in the same group as P1-4 (orange), indicating a duplex allelic status. The three remaining tetraploid parental genotypes Chilean (gray), NECS141 (gray), and F1-192 (red) belong to the other two groups, and likely represent the two simplex genotypes (Fig. 1a). Sequencing of the PCR amplicons of the parental genotypes revealed that a single SNP (T/C) exists at $56 \mathrm{bp}$ of the $111 \mathrm{bp}$ amplicon (Table 1), therefore confirming the hypothesized allelic states based on the melting curve profiles. All five possible allelic states from a single SNP with two variant bases in the parental genotypes can clearly be identified by their melting curve profiles. The two homozygous groups represented by both CC or CCCC (MS-13, MS-186, and Wisfal, blue) and TT (P6-1, green) differ by $0.4^{\circ} \mathrm{C}$ in melting temperature. The CT genotype present in the diploid P1-4 and CCTT genotype corresponding to the 95-608 genotype (orange) represents the broad and short melting curve profile observed in heterozygous genotypes (Fig. 1a, b). The melting curve profile of the Chilean, Altet-4, and NECS-141 genotypes (gray) represents the simplex CTTT genotype and the $x$-axis position of its melting peak is closest to the $\mathrm{T}$ homozygote (green) in the normalized melting peaks plot (Fig. 1a). The CCCT alternative simplex allelic state of the F1-192 genotype (red) has a melting peak closest to the $\mathrm{C}$ homozygote melting peak (blue). In the normalized melting curve plot, the curve of the simplex CTTT is very similar to the melting curves of the duplex (Fig. 1b); however, they are clearly distinguishable in the normalized melting peaks plot (Fig. 1a). Differences in the observed melting curve profiles correspond to the different allelic dosages, e.g. TTTT, CTTT, CCTT, CCCT, and CCCC.

Primer pairs MTTF509 were designed to target the M. truncatula homolog of the LEAFY gene in Arabidopsis, which is a transcription factor regulating flowering time (Yamaguchi et al. 2009), and produce a PCR amplicon $149 \mathrm{bp}$ long. Five melting curve profiles were obtained in the normalized melting peaks (Fig. 1c). The shapes of the peaks suggest two homozygous (gray and blue) and three heterozygous groups (green, red, and orange). In this case, the difference among the heterozygous groups is less than the differences in melting curve profiles obtained using MTTF063. Therefore, it is difficult to determine whether the orange or red melting curve groups represent the duplex genotypes. Sequencing indicates that one $\mathrm{SNP}(\mathrm{A} / \mathrm{G})$ is present at position 

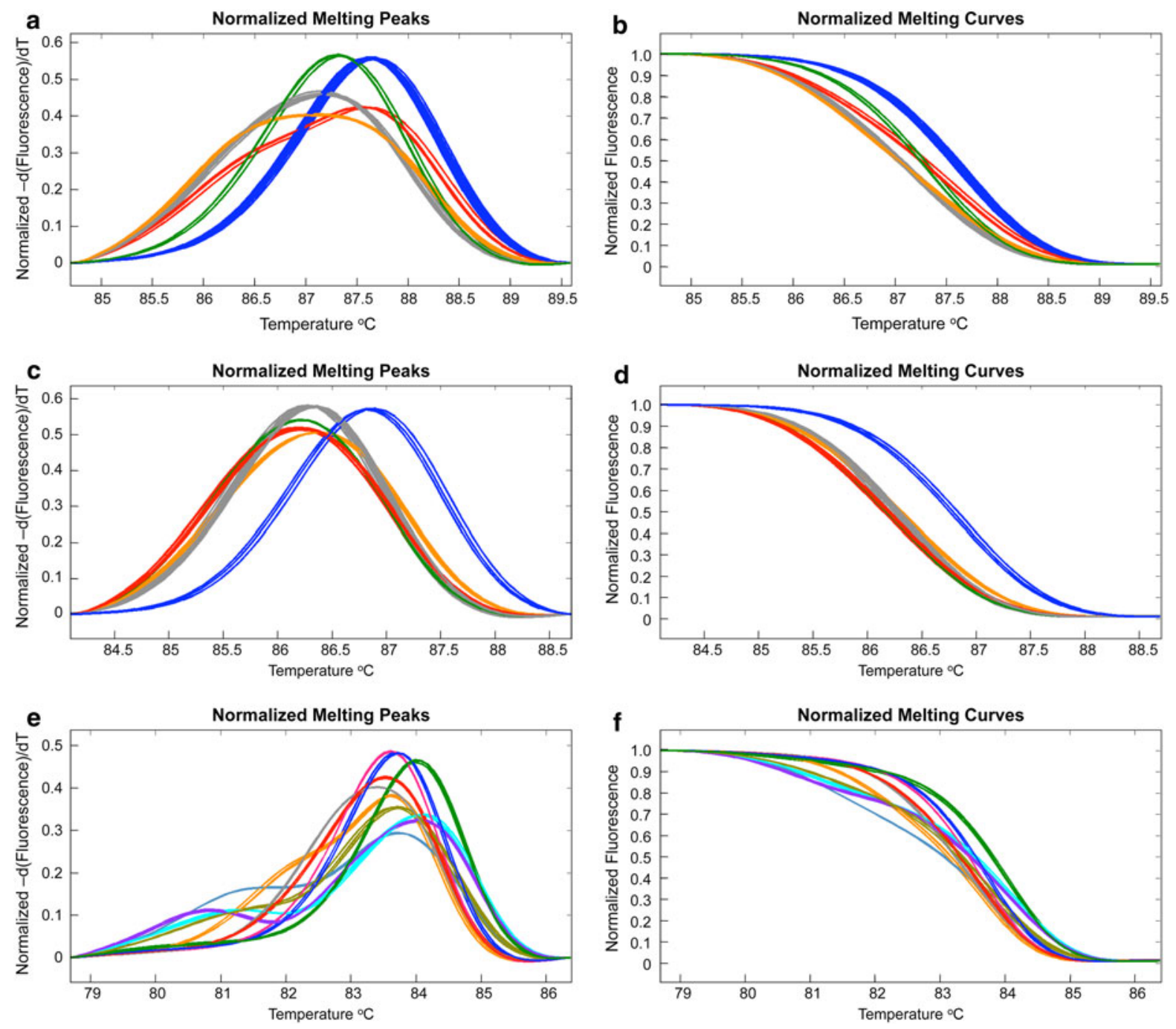

Fig. 1 a and b Small amplicon analysis of ten parents of six alfalfa mapping populations using primer MTTF063 with three replicates. One SNP is present at position 56 in the 111-bp amplicon. Each color represents a different SNP genotype: $(\mathrm{CCCC}=$ blue, $\mathrm{TT}(2 \times)$ and TTTT $(4 \times)=$ green, $\mathrm{CTTT}=$ gray, CT $(2 \times)$ and CCTT $(4 \times)=$ orange, CCCT $=$ red $)$. a Normalized melting peaks of ten mapping parents: P1-4 (orange), P6-1(green), MS-13 (blue), MS-186 (blue), Chilean (gray), Wisfal (blue), F1-192 (red), Altet-4 (gray), 95-608 (orange), and NECS-141 (gray). b Normalized melting curves of ten mapping parents. $\mathbf{c}$ and $\mathbf{d}$ Small amplicon analysis of ten parents of six alfalfa mapping populations using primer MTTF509 with three replicates. One SNP is present at position 123 in the 149-bp amplicon. A second SNP at 79 bp is only present in P6-1. Each color represents a different SNP genotype. At MTTF509 SNP 123, AA $(2 \times)$ and AAAA

$(4 \times)=$ gray, $\quad$ AAAG $=$ green, $\quad$ AG $(2 \times)$ and AAGG $(4 \times)=$ red, $\mathrm{AGGG}=$ orange, $\mathrm{GGGG}=$ blue. At SNP 79, $\mathrm{CCCC}=$ blue and TTTT $=$ all other colors. $\mathbf{c}$ Normalized melting peaks of ten mapping parents: P1-4, MS-13, Chilean and Altet-4 (gray), P6-1 (blue), MS-186, F1-192 and NECS141(red), Wisfal (orange), and 95-608 (green). d Normalized melting curves of ten mapping parents. e and $\mathbf{f}$ Small amplicon analysis of ten parents of six alfalfa mapping populations using primer MTTF562 with three replicates. A total of nine SNPs are present in the parental genotypes. Each color represents a different SNP genotype (Table 2 and Supplementary Table 3). e Normalized melting peaks of ten mapping parents: P1-4 (blue), P6-1 (green), MS-13 (gray), MS-186 (red), Chilean (orange), Wisfal (aqua), F1-192 (light blue), Altet-4 (pink), 95-608 (purple), NECS-141 (light green). f Normalized melting curves of ten mapping parents 
Table 1 Genotypes of parents and progeny of two diploid and four tetraploid alfalfa populations amplified by two SNP primers

\begin{tabular}{|c|c|c|c|}
\hline \multirow[t]{2}{*}{ Population } & \multicolumn{3}{|l|}{ Primers } \\
\hline & MTTF063 & MTTF509 SNP123 & MTTF509 SNP79 \\
\hline \multicolumn{4}{|l|}{ DIP1 } \\
\hline MS-13 & $\mathrm{CC}$ & AA & \\
\hline MS-186 & $\mathrm{CC}$ & $\mathrm{AG}$ & \\
\hline Expected segregation & $\mathrm{CC}$ & AA:AG (1:1) & \\
\hline Progeny segregation (10) & CC (10) & AA:AG (6:4) & \\
\hline \multicolumn{4}{|l|}{ DIP2 } \\
\hline P1-4 & $\mathrm{CT}$ & AA & TT \\
\hline P6-1 & TT & GG & $\mathrm{CC}$ \\
\hline Expected segregation & CT:TT (1:1) & $\mathrm{AG}$ & $\mathrm{CT}$ \\
\hline Progeny segregation (12) & CT:TT $(6: 6=1: 1)$ & $\mathrm{AG}(12)$ & CT (12) \\
\hline \multicolumn{4}{|l|}{$\mathrm{CHBC}$} \\
\hline Chilean & CTTT & AAAA & \\
\hline F1-192 & $\mathrm{CCCT}$ & AAGG & \\
\hline Expected segregation & CTTT:ССТT:CCCT (1:2:1) & AAAA:AAAG:AAGG $(1: 4: 1)$ & \\
\hline Progeny segregation (16) & CTTT:ССТT:СCCT (4:9:3) & AAAA:AAAG:AAGG $(5: 9: 3)$ & \\
\hline \multicolumn{4}{|l|}{ MFBC } \\
\hline Wisfal & $\mathrm{CCCC}$ & AGGG & \\
\hline F1-192 & $\mathrm{CCCT}$ & AAGG & \\
\hline Expected segregation & CCCC:CCCT $(1: 1)$ & AAAG:AAGG:AGGG:GGGG (1:5:5:1) & \\
\hline Progeny segregation (16) & CCCC:CCCT (9:7) & AAGG:AGGG (11:5) & \\
\hline \multicolumn{4}{|l|}{ AL608 } \\
\hline Altet-4 & CTTT & AAAA & \\
\hline NECS-141 & CTTT & AAGG & \\
\hline Expected segregation & CСТT:СТTT:TTTT (1:2:1) & AAAA:AAAG:AAGG $(1: 4: 1)$ & \\
\hline Progeny segregation (16) & ССТT:СТTT:TTTT $(6: 9: 1)$ & AAAA:AAAG:AAGG $(2: 12: 2)$ & \\
\hline \multicolumn{4}{|l|}{ ALNECS } \\
\hline Altet-4 & CTTT & AAAA & \\
\hline $95-608$ & CCTT & AAAG & \\
\hline Expected segregation & СССТ:ССТТ:СТTТ:ТTTТ (1:5:5:1) & AAAA:AAAG (1:1) & \\
\hline Progeny segregation (16) & СССТ:ССТТ:СТTТ (1:9:6) & AAAA:AAAG (7:9) & \\
\hline
\end{tabular}

$123 \mathrm{bp}$ in all the parental genotypes. A second SNP (T/C) at position 79 bp was also identified and P6-1 is the only genotype with the $\mathrm{C}$ variant (Table 1). The gray cluster (Fig. 1c, d) includes the homozygous diploid genotypes MS-13 and P1-4 and the tetraploid genotypes Chilean and Altet-4. The melting temperature differs by $0.5^{\circ} \mathrm{C}$ from the blue homozygous group, which includes the diploid genotype P6-1 with two homozygous SNPs (CC at MTTF509 SNP 79 and GG at MTTF509 SNP 123). Among the three heterozygous groups, one SNP was identified at position $123 \mathrm{bp}$. The simplex AAAG (green) corresponds to 95-608 and has taller peaks than the other two heterozygous. The simplex AGGG (orange) corresponds to Wisfal and can be distinguished from the duplex allelic states AAGG (red) present in F1-192 and NECS-141, based on the position and shape of their corresponding melting peaks. The grouping of individuals into the corresponding allelic dosages was performed based on the normalized melting peaks (Fig. 1c) rather than the normalized melting curves (Fig. 1d).

The primer pairs MTTF562 were designed using the sequence of a $M$. truncatula transcription factor 
encoding a zinc finger ( $\mathrm{C} 2 \mathrm{H} 2$ type) family protein to produce a 146-bp amplicon. Each parental alfalfa genotype has a unique melting curve profile (Fig. 1e, f). The diploid melting curve profiles from P1-4 and P6-1 show that the typical homozygous peaks and the melting curve peaks of MS-13 and MS-186 are wider and shorter, corresponding to their heterozygous allelic composition (Table 2). Sequencing indicates the presence of four SNPs between diploid P1-4 (blue) and P6-1 (green) and each is homozygous at all four positions. There are two SNPs between diploid MS-13 and MS-186. MS-13 is heterozygous $(\mathrm{C} / \mathrm{T})$ at position $44 \mathrm{bp}$ and homozygous (GG) at position 93 bp (gray). MS-186 is homozygous (CC) at position $44 \mathrm{bp}$ and heterozygous (AG) at position $93 \mathrm{bp}$ (red) (Fig. 1e, f and Table 2). The melting curve profiles from the tetraploid parents suggest a complex allelic composition characteristic of multiple SNPs within the amplicon sequence (Fig. 1e, f). The shape of the melting curve profile from Altet-4 suggests a homozygous allelic state at all SNP positions. Sequencing identified seven SNP sites in the ten parental genotypes and Altet-4 is the only one homozygous for all of them (Supplementary Table 3). The remaining tetraploid alfalfa entries evaluated have a unique allelic composition (Supplementary Table 3), each of which corresponds to a distinct melting curve profile (Fig. 1e, f).

\section{SNP marker segregation}

\section{Diploid F1 populations}

In the DIP2 population derived from P1-4 (CT, orange) $\times$ P6-1 (TT, green, Fig. 2a), amplified with MTTF063, the anticipated segregation ratio in the progeny of the two parental genotypes (CT and TT) is 1:1 (Table 1). Six out of the 12 progeny samples evaluated resulted in melting curve profiles corresponding to the parental genotype P1-4, while the other six produced a melting curve profile similar to the parental genotype P6-1 (Fig. 2a), corresponding to the anticipated segregation ratio. Sequencing
Table 2 SNP base positions, genotypes and derived haplotypes of parents and progeny from two diploid alfalfa populations using primer MTTF562

\begin{tabular}{|c|c|c|c|c|c|}
\hline \multirow[t]{2}{*}{ Description } & & \multicolumn{3}{|c|}{ SNP position (bp) } & \multirow{2}{*}{$\begin{array}{l}\text { Genotype based on } \\
\text { the SNP haplotypes }\end{array}$} \\
\hline & & SNP 44 & & SNP 93 & \\
\hline \multicolumn{6}{|c|}{ DIP1 population } \\
\hline \multicolumn{2}{|l|}{ MS-13 } & \multicolumn{2}{|l|}{ CT } & GG & $\mathrm{I}, \mathrm{II}$ \\
\hline MS-186 & & $\mathrm{CC}$ & & AG & I,III \\
\hline \multicolumn{6}{|c|}{ Number of progeny } \\
\hline \multicolumn{2}{|l|}{3} & \multicolumn{2}{|l|}{$\mathrm{CC}$} & AG & $\mathrm{I}, \mathrm{III}=\mathrm{CC} \ldots \mathrm{GA}$ \\
\hline \multicolumn{2}{|l|}{4} & \multicolumn{2}{|l|}{$\mathrm{CC}$} & GG & $\mathrm{I}, \mathrm{I}=\mathrm{CC} \ldots \mathrm{GG}$ \\
\hline \multicolumn{2}{|l|}{1} & \multicolumn{2}{|l|}{ CT } & AG & $\mathrm{II}, \mathrm{III}=\mathrm{TC} \ldots \mathrm{GA}$ \\
\hline \multicolumn{2}{|l|}{2} & \multicolumn{2}{|l|}{ CT } & GG & $\mathrm{I}, \mathrm{II}=\mathrm{CT} \ldots \mathrm{GG}$ \\
\hline \multicolumn{6}{|l|}{ Haplotypes } \\
\hline \multicolumn{2}{|l|}{ I } & \multicolumn{2}{|l|}{$\mathrm{C}$} & $\mathrm{G}$ & \\
\hline II & & \multicolumn{2}{|l|}{$\mathrm{T}$} & G & \\
\hline \multirow[t]{2}{*}{ III } & & \multicolumn{2}{|l|}{$\mathrm{C}$} & A & \\
\hline & SNP 43 & & SNP 66 & SNP 115 & SNP 124 \\
\hline \multicolumn{6}{|c|}{ DIP2 population } \\
\hline P1-4 & AA & & AA & TT & GG \\
\hline P6-1 & TT & & GG & $\mathrm{CC}$ & AA \\
\hline Number of & eny & & & & \\
\hline 12 & $\mathrm{AT}$ & & AG & $\mathrm{TC}$ & $\mathrm{AG}$ \\
\hline Haplotype & & & & & \\
\hline I & A & & A & $\mathrm{T}$ & $\mathrm{G}$ \\
\hline II & $\mathrm{T}$ & & G & $\mathrm{C}$ & A \\
\hline
\end{tabular}



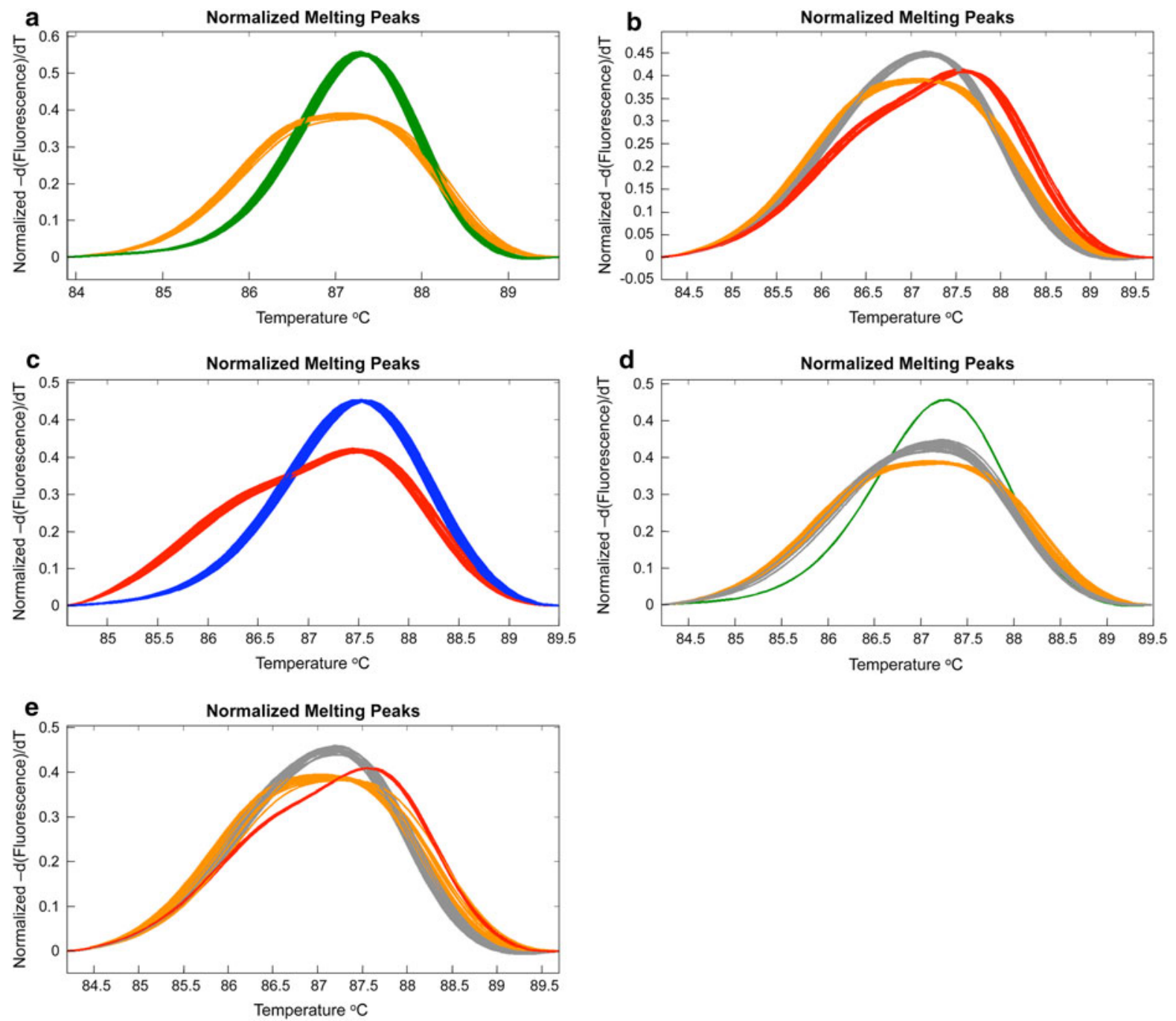

Fig. 2 Normalized melting curves of ten parents and progeny of five alfalfa mapping populations using primer MTTF063 with three replicates. Each color represents a different SNP genotype: $\mathrm{CCCC}=$ blue, TT $(2 \times)$ and TTTT $(4 \times)=$ green, $\mathrm{CTTT}=$ gray, $\quad \mathrm{CT}(2 \times)$ and CCTT $(4 \times)=$ orange, $\mathrm{CCCT}=$ red. a P1-4 (orange) $\times$ P6-1 (green) and 12 progeny (six green and six orange). b Chilean $($ gray $) \times \mathrm{F} 1-192$ (red)

confirmed that all individual diploid F1 progeny with a similar melting curve profile have the same genotype (Table 1). The parental genotypes of the DIP1 population were monomorphic for MTTF063, and therefore no segregation was observed.

In the case of marker MTTF509, the segregation patterns for the allelic classes in the DIP1 population represent the anticipated ratio (Table 1 and Supplementary Fig. 1A). The melting curve profiles from

and 16 progeny (four gray, three red and nine orange). c Wisfal (blue) $\times$ F1-192 (red) and 16 progeny (nine blue and seven red). d Altet-4 (gray) $\times$ NECS-141 (gray) and 16 progeny (one green, six orange and nine gray). e Altet- 4 (gray) $\times$ 95-608 (orange) and 16 progeny (one red, six gray and nine orange)

six F1 progeny correspond to the homozygous (AA) parental genotype from MS-13, shown in gray, while the remaining four progeny correspond to the heterozygous (AG) parental genotype of MS-186, shown in red. For the DIP2 population, both parents are homozygous (P1-4 = AA at MTTF509 SNP 123 and TT at MTTF509 SNP 79, shown in gray, and P6$1=$ GG at MTTF509 SNP 123 and CC at MTTF509 SNP 79, shown in blue; Table 1 and Supplementary 
Fig. 1B), resulting in twelve DIP2 F1 progeny with the same heterozygous genotype at both SNP positions, shown in aqua. Sequencing confirmed the heterozygous nature of all diploid F1 genotypes inferred from their corresponding melting curve profiles.

In the DIP1 population, we identified the segregation of two SNPs corresponding to three haplotypes using marker MTTF562 (Table 2). All four possible genotypes were distinguished using the HRM analysis in the ten DIP1 F1 progeny evaluated (Fig. 3a). In addition to the parental genotypes shown in blue (MS-13) and red (MS-186), two groups corresponding to the combination of the parental alleles, shown in gray and green, were identified. Since the progeny genotypes included a homozygote (I,I) and the heterozygote (II,III), it can be inferred that the gray group represents genotype (I,I) and the green
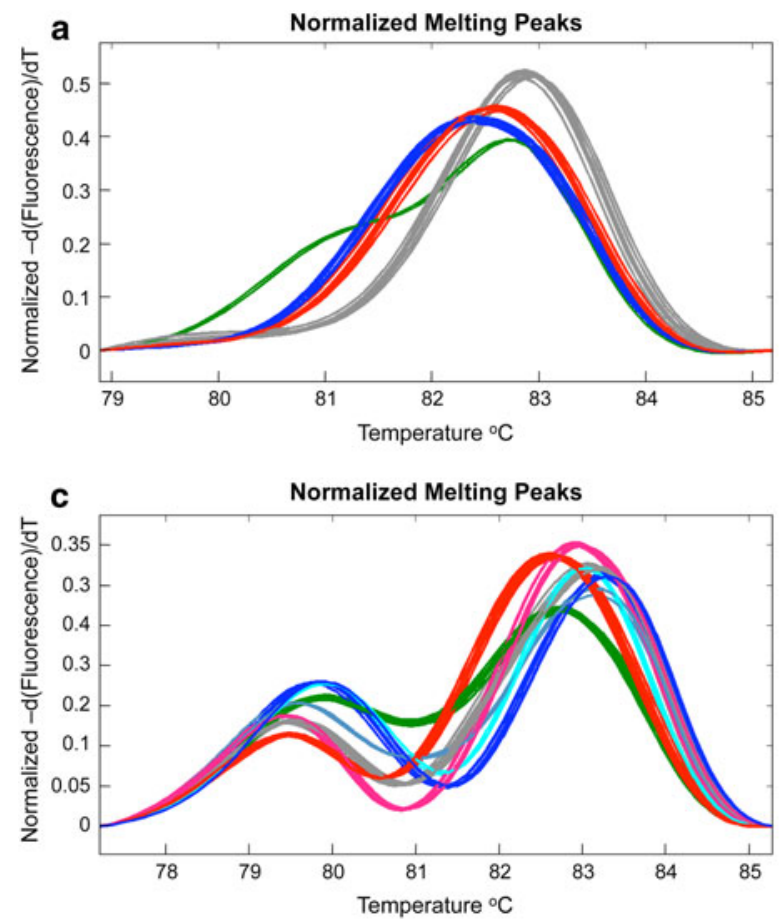

Fig. 3 Normalized melting peaks of six parents and progeny from two diploid and one tetraploid alfalfa mapping populations using primer MTTF562 with three replicates. The genotypes are shown based on haplotype (Table 2 and Supplementary Table 4). a DIP1 population: MS-13 (blue; genotype I,II) $\times$ MS-186 (red = genotype I,III) and 10 progeny (one green = genotype II,III; two blue = genotype I,II; three red = genotype I,III; and four gray = genotype I,I). b DIP2 represents genotype (II,III) based on the shape of the melting peaks. Sequencing confirmed that the haplotype combinations for all groups shown in gray, blue, red, and green are (I,I), (I,II), (I,III), and (II,III), respectively (Table 2). In the DIP2 population (Fig. 3b), there are four SNPs which are homozygous in each parent. Therefore, all F1 progeny are heterozygous at all loci, as shown in the single group of melting peaks (gray) and by sequencing (Table 2).

\section{Tetraploid backcross populations}

Molecular markers useful for mapping in tetraploid alfalfa backcross populations (CHBC and MFBC) are those segregating as single-dose markers (AAAA vs. GAAA) with an expected 1:1 segregation ratio (Sledge et al. 2005). In the tetraploid MFBC population, the $(\mathrm{C} / \mathrm{T})$ SNP from MTTF063 is segregating

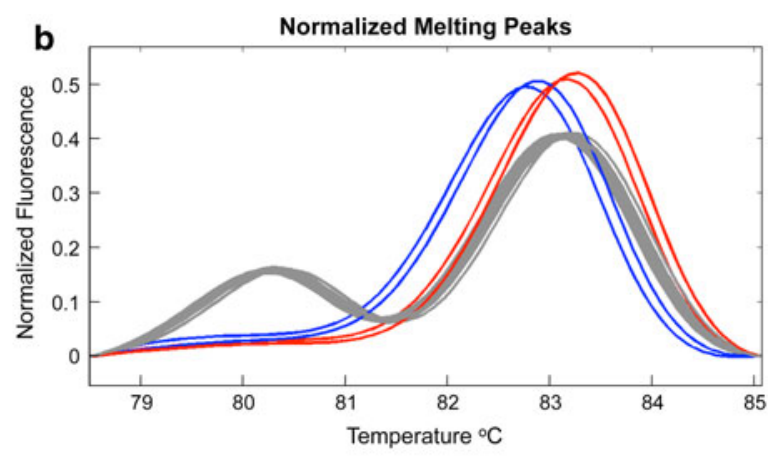

population, P1-4 (blue = genotype I,I) $\times$ P6-1 $($ red $=$ genotype II,II) and 12 progeny (gray = genotype I,II). c MFBC population, Wisfal $($ gray = genotype $\mathrm{I}, \mathrm{II}, \mathrm{IV}, \mathrm{V}) \times \mathrm{F} 1-192$ $($ green $=$ genotype $\mathrm{I}, \mathrm{III}, \mathrm{IV}, \mathrm{V})$ and 16 progeny $($ four red $=$ genotype I,I,IV,V; three gray= genotype I,II,IV,V; three green = genotype I,III,IV,V; two blue = genotype II,III,IV,V; two pink = genotype I,I,V,V, one aqua = genotype I,III,V,V; and one light blue = genotype II,III,IV,IV) 
in a single-dose allele pattern (Table 1 and Fig. 2c). The cross between the homozygote Wisfal (CCCC, blue) and the simplex F1-192 (CCCT) resulted in a 1:1 segregation of the two parental genotypes in the progeny. In the melting analysis, nine out of 16 progeny clustered with the Wisfal parent (blue) and the other seven were grouped with the F1-192 (red). The sequencing results confirm the SNP composition identified by the melting curve profiles. Genotyping 93 individuals from the MFBC population identified 58 individuals with the CCCC melting profile and 35 individuals with the CCCT melting profile. Mapping using JoinMap resulted in the identification of MTTF063 to linkage group 2 (Supplementary Table 2). In the CHBC backcross population, the cross was between Chilean (CTTT), shown in gray, and F1192 (CCCT), shown in red (Fig. 2b and Table 1). Therefore, the expected segregation was 1:2:1 corresponding to genotypes CTTT, CCTT, and CCCT, which are clearly separated in the melting curve profiles. In addition to the two parental groups, a third group of melting curves shows the same profile as P1-4 (CT, Fig. 2a), suggesting that the genotype for these individuals was CCTT. The proposed allelic status of these individuals was confirmed by sequencing.

In the MFBC population, the expected segregation pattern for MTTF509 SNP 123 was AAAG, AAGG, AGGG, GGGG with a 1:5:5:1 ratio (Table 1). In the 16 progeny evaluated, we only detected the two parental genotypes (Supplementary Fig. 1C). In the CHBC population, it was possible to distinguish the three expected genotypes AAAA, AAAG, and AAGG based on their distinct melting peaks (Supplementary Fig. 1D). Therefore, the absence of the other two genotypes in the MFBC population is a result of their anticipated low numbers in the progeny and the limited number of progeny from the MFBC population initially evaluated rather than due to a limitation in the assay.

Multiple SNP were detected among the tetraploid parents using primer MTTF562 (Supplementary Table 3). The segregation of these SNPs in the progeny resulted in the combination of SNP alleles and thus a large number of possible genotypes. The parental genotypes and 16 MFBC backcross progeny represent an example of the SNP haplotype analysis (Supplementary Table 4). Based on the sequence data, five haplotypes and seven genotypes were identified. Although the segregation pattern is more complex that in the case of one or two SNP, each genotype was represented by a distinct melting curve profile (Fig. 3c).

\section{Tetraploid F1 mapping populations}

Simplex, duplex, and double simplex markers are the types of segregation patterns for markers which can be mapped using TetraploidMap (Hackett and Luo 2003). Translated into SNP genotypes, a simplex marker (e.g. AGGG $\times$ GGGG) will segregate in a 1:1 ratio between the two parental genotypes. A duplex marker (e.g. AAGG $\times$ GGGG) segregates in the combinations AAGG, AGGG, GGGG in a 1:4:1 ratio, respectively. The progeny can be grouped as AAGG + AGGG:GGGG with a 5:1 segregation ratio for mapping. Double simplex markers (e.g. AGG$\mathrm{G} \times \mathrm{AGGG)}$ segregate in a 1:2:1 ratio, and correspond to AAGG, AGGG, GGGG. In this case, the progeny can be grouped as AAGG + AGGG:GGGG with a 3:1 segregation ratio for mapping. In the AL608 population, MTTF063 represents the double simplex marker CTTT $\times$ CTTT $($ Table 1$)$. The three expected segregating genotypes CCTT, CTTT, and TTTT were identified based on the observed melting peaks (Fig. 2d) and correspond with the sequencing results (Table 1). Based on the shape of the HRM peaks, it was possible to identify the two non-parental segregants represented in the progeny, including the homozygote TTTT (green) and the CCTT heterozygote (orange). In the ALNECS population, marker MTTF063 displayed the simplex CTTT in Altet-4 and the duplex CCTT composition in 95-608 (Table 1). The expected segregation pattern is 1:5:5:1 for the genotypes CCCT, CCTT, CTTT, TTTT, respectively. The three heterozygous genotypes were observed in the melting curve profiles from the $16 \mathrm{~F} 1$ progeny evaluated (Fig. 2e).

MTTF509 in the AL608 population represents a duplex marker with Altet-4 corresponding to AAAA and NECS-141 to AAGG. The possible combinations are AAAA, AAAG, AAGG which are segregating in the ratio $1: 4: 1$ in the progeny. The 16 progeny evaluated resulted in three distinct melting peak profiles (Supplementary Fig. 1E). The three groups were distinguished by the height and relative position of the melting peaks (AAAA > AAAG > AAGG). In the ALNECS population, MTTF509 represents a simplex marker between Altet-4 (AAAA) and 95-608 (AAAG) with an expected 
AAAA, AAAG segregation ratio of 1:1. Seven progeny evaluated correspond to the AAAA allelic status and nine possess the AAAG combination. HRM genotyping in 185 individuals from the ALNECS population was used to map MTTF509 to linkage group 3 (Supplementary Table 2).

A total of 14 SNP markers were mapped in the two backcross populations and 13 SNP markers were mapped in the ALNECS F1 population (Supplementary Table 2). At least one SNP marker was mapped to each of the eight linkage groups in the backcross populations. Eight markers were mapped in both populations and they mapped to the same linkage groups (Supplementary Table 2 and Supplementary Fig. 2). The position, marker order, and relative distance were similar in the two population types and correspond to similar positions on the M. truncatula chromosome 2.

\section{Discussion}

Requirements for SNP genotyping platforms in polyploid species include allelic dosage sensitivity and detection of haplotype combinations in heterozygous genotypes. SNP detection technologies have evolved from expensive, time-consuming, and laborintensive processes to some of the most highly automated, efficient, and relatively inexpensive methods of DNA marker detection (Kwok and Chen 2003). In HRM assays, differences in the melting curve shape between amplicons indicate that the DNA samples differ in sequence composition within the amplicon (Liew et al. 2004). Melting acquisition and analysis can be performed in less than $10 \mathrm{~min}$ after PCR, and is a closed-tube system which does not require post-PCR sample processing. Some genotyping techniques require fluorescently labeled probes which are costly and capable of detecting only a single allele (Montgomery et al. 2007). In contrast, genotyping by HRM does not require expensive fluorescent labels and multiple alleles can be detected in a single amplicon. Additionally, no previous knowledge of the possible haplotypes present in a genotype is required (De Koeyer et al. 2010). An additional advantage of HRM analysis over probe or oligonucleotide array-based systems is the flexibility in adding or replacing SNP markers of interest after establishing the system.
Small amplicon genotyping with temperature controls increases the sensitivity of the amplicon genotyping and allows better discrimination of the samples (Seipp et al. 2007). In this study, HRM small amplicon assays were developed for three candidate loci targeting transcription factor sequences and showing different patterns of segregation complexity (Supplementary Table 2). The resulting melting curve profiles from all replications in the small amplicon genotyping assay using temperature calibrators consistently identified SNP and distinguished allelic dosage both at the diploid and tetraploid level. Therefore, technical replications are not needed for SNP discovery, genotyping, and mapping. The effort to avoid amplification of multiple genomic regions during the primer design process resulted in the specificity and sensitivity to distinguish each genotype. Although differences in allelic dosage are visible in the normalized melting curve plots, the sensitivity of the assay is most clearly visualized in the normalized melting peak plots. In this study, small amplicon analysis using HRM proved to be a sensitive and consistent platform for SNP genotyping in both diploid and tetraploid alfalfa with amplicon sizes suitable for HRM. Wu et al. (2009) was able to achieve SNP detection via HRM with amplicon sizes close to $100 \mathrm{bp}$ while De Koeyer et al. (2010) reported success with HRM for SNP genotyping in 50- to 230-bp amplicons.

These results show the feasibility of using small amplicon HRM analysis for SNP identification and estimation of allelic dosage in polyploid species without prior sequence information. The HRM pipeline involves evaluating differences in the melting curve profiles of the parental genotypes, followed by screening of the polymorphic markers with a subset of individuals from the population to evaluate segregation patterns. As shown in this study, SNP markers suitable for diploid and tetraploid mapping based on segregation patterns can be identified using HRM. In plants, amplicon melting has been used for mapping SNP markers in soybean (Monteros et al. 2010), white lupin (Croxford et al. 2008), and apple (Chagné et al. 2008). Our mapping efforts using HRM successfully added markers to linkage maps developed from both backcross and F1 tetraploid alfalfa mapping populations. The grouping of individuals based on melting curve profile was amenable to reliable semi-automated allele calling. We are 
working to enhance marker coverage in the alfalfa mapping populations using HRM targeting genomic regions of interest and gaps currently in SSR-based linkage maps (Sledge et al. 2005; Khu et al. 2010).

HRM analysis of PCR amplicons is a powerful method for detecting sequence variations at any position within an amplicon. It is a simple and versatile method for SNP genotyping that does not require application of the PCR products onto a gel or other matrix to separate and detect sequence variation (Montgomery et al. 2007). The advantages of HRM analysis over other SNP genotyping methods is the dosage sensitivity, ability to detect high levels of heterozygosity, and flexibility in adding or replacing SNP markers with only the cost of primer development, without the need to develop an entire array. As our knowledge of the genes affecting relevant traits in crop plants and knowledge of their corresponding sequence variation increases due to advances in genomics and mapping studies, HRM represents an ideal platform for SNP identification and genotyping and enables implementation of molecular breeding approaches in polyploid species.

\section{Conclusion}

We report the utilization of high-resolution melting analysis using the small amplicon assay to identify SNP variation and assay allelic dosage in diploid and tetraploid alfalfa. The approach described represents an efficient platform for SNP detection and genotyping in diploid and tetraploid species. HRM analysis can be readily adapted for large-scale genotyping and mapping of SNP markers in any species with limited genomic resources. The ability to detect allelic dosage at each SNP locus in tetraploid species represents a significant advance addressing a practical limitation for SNP genotyping in polyploids. The availability of improved platforms for SNP genotyping and polymorphism detection facilitates comparative mapping studies and integration of genome-wide selection and molecular breeding strategies aimed at improving the efficiency of breeding programs.

Acknowledgments This research was supported by The Samuel Roberts Noble Foundation, Inc. and Forage Genetics International. We would like to thank E. Charles Brummer for providing samples of the diploid Medicago sativa subsp. caerulea MS-186 and MS-13, and the corresponding F1 progeny. We also thank Drs. Mark Sorrells and Malay Saha for providing valuable suggestions to the manuscript.

Open Access This article is distributed under the terms of the Creative Commons Attribution Noncommercial License which permits any noncommercial use, distribution, and reproduction in any medium, provided the original author(s) and source are credited.

\section{References}

Chagné D, Gasic K, Crowhurst RN, Han Y, Bassett HC, Bowatte DR, Lawrence TJ, Rikkerink EHA, Gardiner SE, Korban SS (2008) Development of a set of SNP markers present in expressed genes of the apple. Genomics 92: 353-358

Cho MH, Ciulla D, Klanderman BJ, Raby BA, Silverman EK (2008) High-resolution melting curve analysis of genomic and whole-genome amplified DNA. Clin Chem 54: 2055-2058

Croxford AE, Rogers T, Caligari PDS, Wilkinson MJ (2008) High-resolution melt analysis to identify and map sequence-tagged site anchor points onto linkage maps: a white lupin (Lupinus albus) map as an exemplar. New Phytol 180:594-607

De Koeyer D, Douglass K, Murphy A, Whitney S, Nolan L, Song Y, De Jong W (2010) Application of high-resolution DNA melting for genotyping and variant scanning of diploid and autotetraploid potato. Mol Breed 25:67-90

Gallais A (2003) Quantitative genetics and breeding methods in autopolyploid plants. INRA, Paris, France

Ha BK, Boerma HR (2008) High-throughput SNP genotyping by melting curve analysis for resistance to southern rootknot nematode and frogeye leaf spot in soybean. J Crop Sci Biotech 11:91-100

Hackett CA, Luo ZW (2003) TetraploidMap: construction of a linkage map in autotetraploid species. J Hered 94: 358-359

Han Y, Kang Y, Torres-Jerez I, Cheung F, Town CD, Zhao PX, Udvardi MK, Monteros MJ (2010) Genome-wide SNP discovery, validation and mapping in tetraploid alfalfa. In: Proceedings of the Vth international congress on legume genetics and genomics, 2-8 July. Asilomar, CA, p 23

Hurley JD, Engle LJ, Davis JT, Welsh AM, Landers JE (2005) A simple, bead-based approach for multi-SNP molecular haplotyping. Nucleic Acids Res 32:e186

Kakar K, Wandrey M, Czechowski T, Gaertner T, Scheible WR, Stitt M, Torres-Jerez I, Xiao Y, Redman JC, Wu HC, Cheung F, Town CD, Udvardi MK (2008) A community resource for high-throughput quantitative RT-PCR analysis of transcription factor gene expression in Medicago truncatula. Plant Methods 4:18

Khu DM, Reyno R, Brummer EC, Bouton JH, Monteros MJ (2010) QTL mapping of aluminum tolerance in tetraploid 
alfalfa. In: Huyghe C (ed) Sustainable use of genetic diversity in forage and turf breeding. Springer, Netherlands, pp 437-442

Kwok PY (2001) Methods for genotyping single nucleotide polymorphisms. Annu Rev Genomics Hum Genet 2: 235-258

Kwok PY, Chen X (2003) Detection of single nucleotide polymorphisms. Curr Issues Mol Biol 5:43-60

Liew M, Pryor R, Palais R, Meadows C, Erali M, Lyon E, Wittwer C (2004) Genotyping of single-nucleotide polymorphisms by high-resolution melting of small amplicons. Clin Chem 50:1156-1164

McCoy TJ, Bingham ET (1988) Cytology and cytogenetics of alfalfa. In: Hanson AA, Barnes DK, Hill RR (eds) Alfalfa and alfalfa improvement. American Society of Agronomy, Madison, pp 737-776

Monteros MJ, Ha BK, Boerma HR (2010) SNP assay to detect the 'Hyuuga' red-brown lesion resistance gene for Asian soybean rust. Theor Appl Genet 121:1023-1032

Montgomery J, Wittwer CT, Palais R, Zhou L (2007) Simultaneous mutation scanning and genotyping by high-resolution DNA melting analysis. Nat Protocols 2:59-66

Neigenfind J, Gyetvai G, Basekow R, Diehl S, Achenbach U, Gebhardt C, Selbig J, Kersten B (2008) Haplotype inference from unphased SNP data in heterozygous polyploids based on SAT. BMC Genomics 9:356

Reed GH, Wittwer CT (2004) Sensitivity and specificity of single-nucleotide polymorphism scanning by high-resolution melting analysis. Clin Chem 50:1748-1754

Seipp MT, Durtschi JD, Liew MA, Williams J, Damjanovich K, Pont-Kingdon G, Lyon E, Voelkerding KV, Wittwer CT (2007) Unlabeled oligonucleotides as internal temperature controls for genotyping by amplicon melting. J Mol Diagn 9:284-289

Sledge MK, Ray IM, Jiang G (2005) An expressed sequence tag SSR map of tetraploid alfalfa (Medicago sativa L.). Theor Appl Genet 111:980-992
Smith GD, Chadwick BE, Willmore-Payne C, Bentz JS (2008) Detection of epidermal growth factor receptor gene mutations in cytology specimens from patients with nonsmall cell lung cancer utilising high-resolution melting amplicon analysis. J Clin Pathol 61:487-493

Soltis DE, Soltis PS (1995) The dynamic nature of polyploid genomes. Proc Nat Acad Sci USA 92:8089-8091

Studer B, Jensen L, Fiil A, Asp T (2009) "Blind" mapping of genic DNA sequence polymorphisms in Lolium perenne L. by high resolution melting curve analysis. Mol Breed 24:191-199

Taylor CF (2009) Mutation scanning using high-resolution melting. Biochem Soc Trans 37:433-437

Van Ooijen JW, Voorrips RE (2001) JoinMap ${ }^{\circledR}$ 3.0. Software for the calculation of genetic linkage maps. Plant Research International, Wageningen, Netherlands

Wang X, Brummer EC (2010) Developing a reference genetic map for alfalfa and mapping of biofuel traits. In: Abstracts of the plant and animal genomes XVIII conference, San Diego

Wu SB, Tavassolian I, Rabiei G, Hunt P, Wirthensohn M, Gibson J, Ford C, Sedgley M (2009) Mapping SNPanchored genes using high-resolution melting analysis in almond. Mol Genet Genomics 282:273-281

Yamaguchi A, Wu M-F, Yang L, Wu G, Poethig RS, Wagner D (2009) The microRNA-regulated SBP-Box transcription factor SPL3 is a direct upstream activator of LEAFY, FRUITFULL, and APETALA1. Dev Cell 17:268-278

Zhang Y, He J, Zhao P, Bouton J, Monteros M (2008) Genome-wide identification of microsatellites in white clover (Trifolium repens L.) using FIASCO and phpSSRMiner. Plant Methods 4:19

Zhou L, Myers AN, Vandersteen JG, Wang L, Wittwer CT (2004) Closed-tube genotyping with unlabeled oligonucleotide probes and a saturating DNA dye. Clin Chem 50:1328-1335 